\title{
Tag-free Combinators for Binding-Time Polymorphic Program Generation
}

\author{
Peter Thiemann ${ }^{1}$ and Martin Sulzmann ${ }^{2}$ \\ 1 Albert-Ludwigs-Universität Freiburg, Germany \\ thiemann@informatik.uni-freiburg.de \\ 2 Intaris martin.sulzmann@gmail.com
}

\begin{abstract}
Binding-time polymorphism enables a highly flexible bindingtime analysis for offline partial evaluation. This work provides the tools to translate this flexibility into efficient program specialization in the context of a polymorphic language.

Following the cogen-combinator approach, a set of combinators is defined in Haskell that enables the straightforward transcription of a bindingtime polymorphic annotated program into the corresponding program generator. The typing of the combinators mimics the constraints of the binding-time analysis. The resulting program generator is safe, tag-free, and it has no interpretive overhead.
\end{abstract}

\section{Introduction}

A polymorphic binding-time analysis empowers an offline partial evaluator to obtain specialization results on par with those of an online partial evaluator. However, implemented specializers for polymorphic binding-time analysis so far do not exploit the efficiency potential of offline partial evaluation. They are interpreter-based, they pass and interpret binding-time descriptions at specialization time, and they use tagging to distinguish ordinary static values from dynamic values (generated code).

For monomorphic binding-time analysis, there is a well-known approach to obtain compiled, tag-free program generators that perform offline partial evaluation. The cogen approach to partial evaluation [13] explains the direct construction of a program generator from a binding-time annotated program. For typed languages, this direct generation step is more efficient than going via the Futamura projections, which can lead to multiple levels of data encoding [11].

For example, the binding-time annotated power function

$$
\text { power } x^{D} n^{S}=\text { if } n={ }^{S} 0 \text { then lift } 1 \text { else } x *{ }^{D} \text { power } x\left(n-{ }^{S} 1\right)
$$

uses the superscripts ${ }^{S}$ and ${ }^{D}$ to indicate static and dynamic operations that happen at specialization time and at run time, respectively. The lift expression avoids a binding-time mismatch by converting the static constant 1 into the required dynamic code at that point. The translation to a program generator can be done in a compositional way, by specifying a translation for each annotated syntactic construct: The constructs annotated with ${ }^{S}$ are translated to 
themselves, the constructs annotated with ${ }^{D}$ are translated to expressions that generate the respective expression tree, and lift maps to the appropriate syntax constructor. A translation to Haskell would look like this:

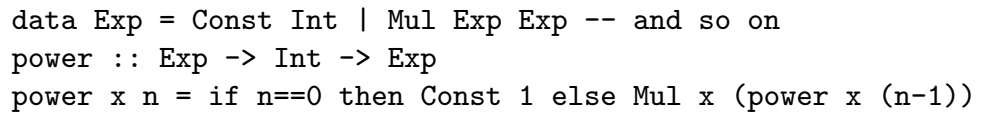

This simple example already demonstrates that the static data is neither encoded nor tagged and that, consequently, the static expressions execute efficiently.

The methods used so far for translating binding-time annotated programs to program generators are only suitable for monovariant annotation schemes [1,2, $18,20]$. They do not cover annotations created by the more precise polyvariant binding-time analyses [5, 6, 8-10]. A polyvariant binding-time analysis enables abstraction over concrete binding times. To continue the example, the power function would receive three additional binding-time parameters that express the binding times of the arguments $x$ and $n$ and of the result of power, which must be more dynamic than either argument:

$$
\begin{aligned}
(\text { power }:: & \text { Int } \rightarrow \text { Int } \rightarrow \text { Int }): \forall \beta \gamma \delta .(\beta \leq \delta, \gamma \leq \delta) \Rightarrow \beta \stackrel{S}{\mapsto} \gamma \stackrel{S}{\mapsto} \delta \\
\text { power } \beta \gamma \delta x n= & \text { if } n={ }^{\gamma} \text { lift }^{S, \gamma} 0 \text { then } \text { lift }^{S, \delta} 1 \\
& \text { else } \text { lift }^{\beta, \delta} x *^{\delta} \text { power } \beta \gamma x\left(n-\gamma{ }^{\gamma} \text { lift }^{S, \gamma} 1\right)
\end{aligned}
$$

Evidently, the lift expression must be generalized to lift ${ }^{\beta, \delta}$ which converts a base-type value of binding time $\beta$ to binding time $\delta$. This conversion requires $\beta \leq \delta$ where the ordering is the least partial order such that $S \leq D$. The other constraint, $\gamma \leq \delta$, arises from the conditional. The binding time $\gamma$ of the condition is a lower bound of the binding time $\delta$ of the result.

The translation of this annotated program to a satisfactory program generator becomes more tricky. Fig. 1 shows the naive approach, which is hardly satisfactory. First, binding times have to be passed and tested explicitly in the generator. Second, the generator relies on run-time tags to identify static and dynamic values in the Value datatype as evident from the implementations of pIf and p0p2: An untagged generator could omit stripping off (and reapplying) the Bool and Exp tags. Indeed, the BT argument would not be needed for the pIf combinator. ${ }^{3}$ Third, the generator is not self-checking. Its type does not incorporate the constraints from the binding-time annotation, thus it can give rise to run-time errors because of binding-time mismatches. For example, an invocation (powergen D D S) can result in a run-time error when attempting to execute (pLift D $\mathrm{S} x$ ).

This particular generator has further shortcomings not addressed in this work. For example, (powergen D D D $\mathrm{x}$ ) does not terminate, for any $\mathrm{x}$ and

\footnotetext{
${ }^{3}$ Use of the combinator still requires a preceding binding-time analysis because it expects its e1 and e2 arguments to carry the Exp tag if the condition carries an Exp tag. Unlike the staged interpreters of Carette and coworkers [3], this pIf combinator would not be suitable for online partial evaluation because the dynamic version of the conditional does not convert static values in the branches to dynamic values.
} 


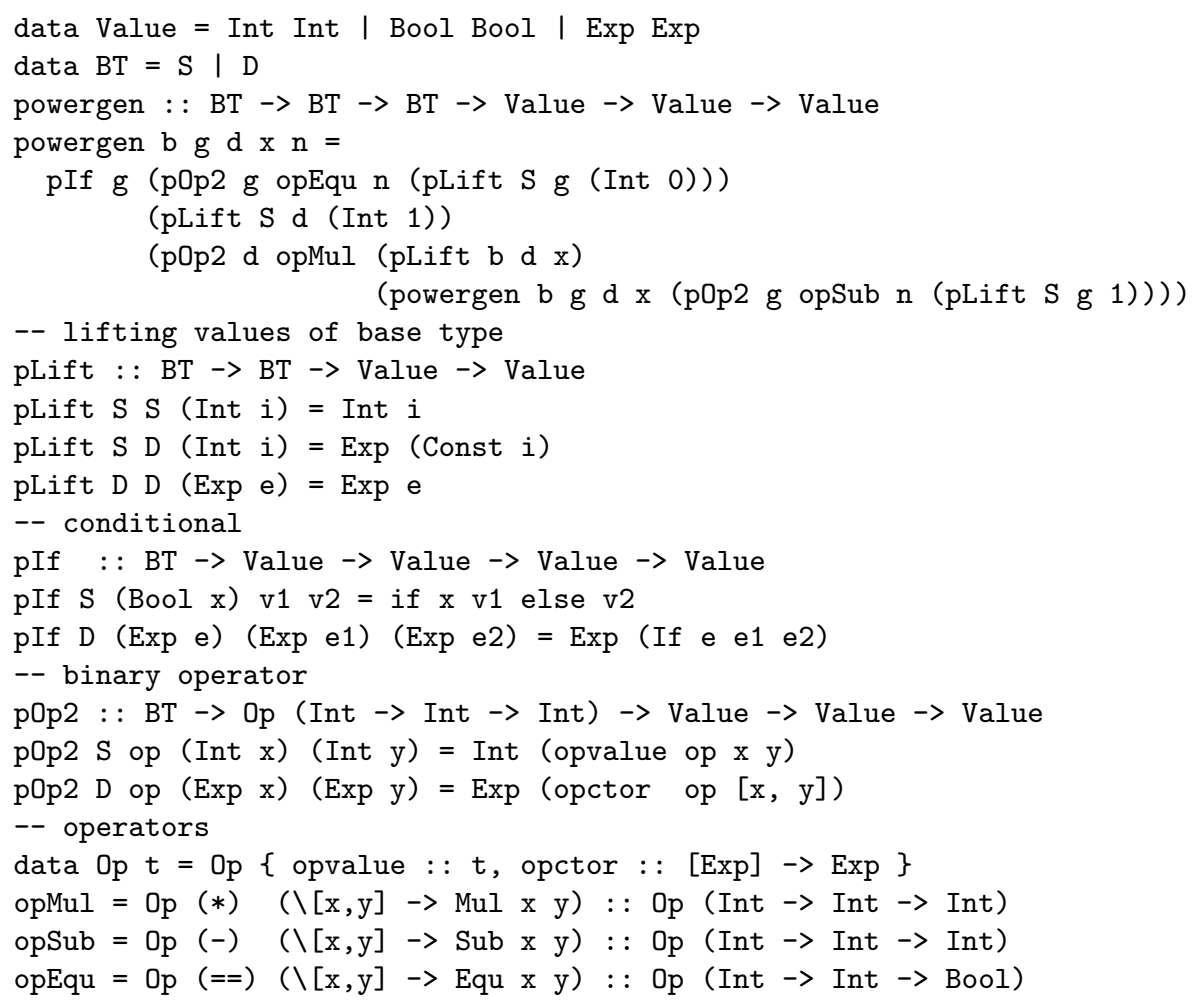

Fig. 1. Naive generator with binding-time polymorphism.

$\mathrm{n}$, because the recursive call to powergen is implicitly static, that is, it is always performed at specialization time.

The present work is the first to address the construction of efficient program generators with polymorphic binding times. Because it applies to languages with ML-style polymorphism, it paves the way for efficient program specialization for Haskell. It addresses all shortcomings of the naive generator.

1. No interpretive overhead. Binding-time descriptions are passed at run time but they are never tested. Due to laziness they have virtually no cost.

2. Tag-free. The generator requires no tagging, neither type tags nor tags to distinguish static from dynamic values.

3. Safety. The typing of the generator ensures that binding-time inconsistencies in the input of the generator are caught by the type checker before starting the specialization.

The main contribution is a set of combinators that enables the construction of tag-free program generators via a simple type-directed translation from a polymorphic binding-time type derivation to a Haskell program using these combinators. The starting point is the polyvariant binding-time analysis for ML-style polymorphic languages by Glynn and coworkers [6]. 


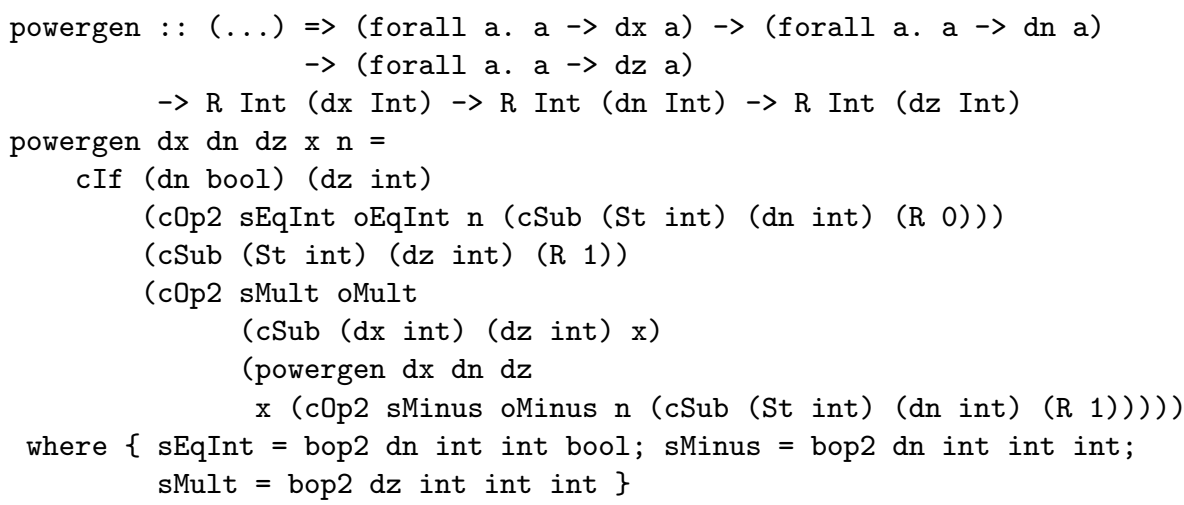

Fig. 2. Tagfree generator for specializations of power. The type signature is truncated to save space.

The implementation is in Haskell [15] with various extensions (e.g., type functions [17], multi parameter type classes, rank-2 types [16], GADTs) as implemented by GHC. For lack of space, we assume familiarity with the language and the extensions.

\section{Tagfree Polymorphic Program Generation}

Figure 2 contains the tag free variant of the polymorphic generator for the power function shown in Fig. 1. Before delving into a detailed explanation of the combinators, let's introduce some preliminaries and run the generator on examples.

Like the previous generator, the new generator receives three binding-time parameters and two value parameters. Binding times are represented by polymorphic functions that construct binding-time descriptions (bt descriptions), which are passed to the combinators. A bt description has the same structure as the underlying type but alternates binding times with regular type constructors. Binding times are represented by two data types, St and Dy.

newtype St a = St a -- static annotation

newtype Dy a = Dy a -- dynamic annotation

For example, (St int : : St Int) describes a static integer and (St (St Int $\rightarrow$ Dy Bool)) is the type of a description of a static function with static input and dynamic output. Descriptions are reified type arguments, which are never evaluated.

Depending on the instantiation of the binding time parameters, powergen exhibits dramatically different behaviors as shown and labeled in Fig. 3. The nontermination of the third example is the expected behavior because the recursion in powergen is always static. The error message for the last example accurately reflects the failing constraints of the binding-time analysis $(\S 2.1, \S 2.3)$.

The computation of the generator happens in terms of a representation type $\mathrm{R} t$ btd, which depends on the underlying type $t$ and its bt description btd. 


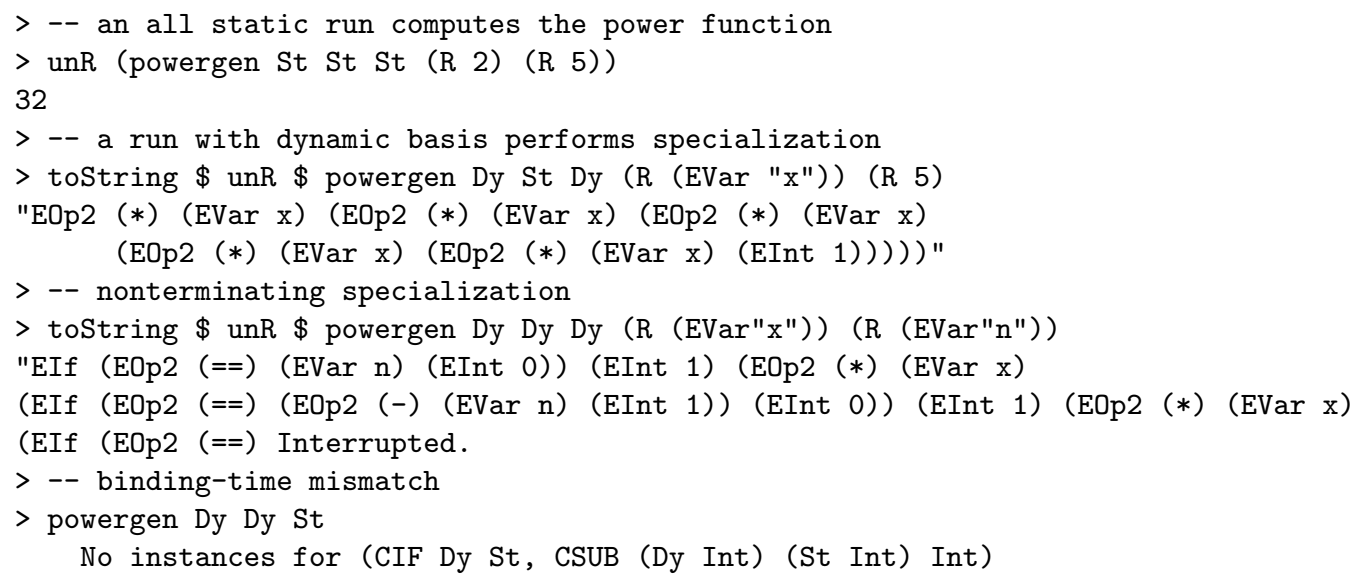

Fig. 3. Running powergen.

Any value that is passed into (out of) the generator must first be wrapped (unwrapped). As $\mathrm{R}$ is an isomorphism, its use does not amount to tagging. ${ }^{4}$

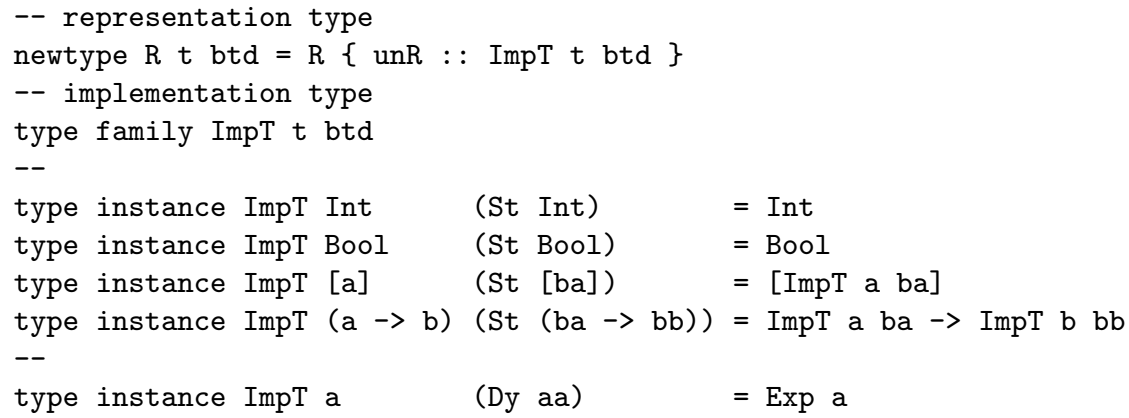

The argument to the $\mathrm{R}$ constructor must have the implementation type, computed by the type function ImpT. For type constructors with static bt descriptions, ImpT rebuilds the type constructors and translates components of the type recursively. This strategy implies that static computations are implemented by themselves. If the translation hits a dynamic annotation, then well-formedness dictates that further components of the type carry a dynamic annotation, too. Hence, any value of dynamic type a is implemented as an expression of type Exp a. The latter type is a GADT with the usual definition (see appendix).

\subsection{Basic Combinators}

Continuing the analysis of the code in Fig. 2, the cIf combinator takes two bt descriptions, one ( $\mathrm{dn}$ bool) describing the binding time of the condition and one

\footnotetext{
${ }^{4}$ The reader may wonder why $\mathrm{R} t$ btd is needed as it is isomorphic to $\operatorname{Imp} T \mathrm{t}$ btd. However, when type inference equates $\mathrm{R} t 1 \mathrm{~b} 1=\mathrm{R}$ t2 $\mathrm{b} 2$ it can deduce that $\mathrm{t} 1=$ t2 and $\mathrm{b} 1=\mathrm{b} 2$. It cannot deduce these equalities from $\operatorname{ImpT}$ t1 b1 = ImpT t2 b2.
} 
( $d z$ int) fixing the binding time of the result of the conditional. The remaining arguments stand for the condition, the true-branch, and the false-branch, where the two branches have the same representation type. cIf is overloaded such that there are instances for either static dn and arbitrary $d z$ or for dynamic $d n$ and $d z$. The type checker rejects any other combination of binding times (via unresolved instance), thus enforcing the constraints of the binding-time analysis. The definition shows that the bt descriptions are not touched.

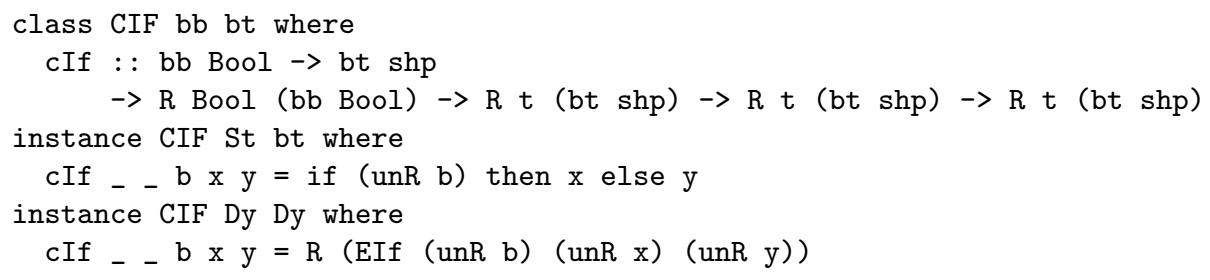

The combinator c0p2 for binary primitive operations takes a bt description for the type of the operation, the operation itself, and its two arguments. It is implemented in terms of a more general operator cConst, which injects constants into a generator, and function application cApp. Again, the overloading of these combinators enables the dual use of static and dynamic operations.

data Op a $=0 p\{$ opname : : String, opvalue : : a $\}$

c0p2 btd op $x$ y $=$ cApp undefined (cApp btd (cConst btd op) $x$ ) y

Instead of examining the unwieldy type of c0p2, it is simpler and more general to look at the cConst operator (but see Appendix C). To safely embed a constant of arbitrary type in a generator requires that the constant's bt description is uniform, that is, it is either completely static or completely dynamic [19]. This requirement is stronger than the usual well-formedness (see $\S 3.4$ ), which can be enforced locally. Uniformity is asserted with a two-parameter type class Uniform.

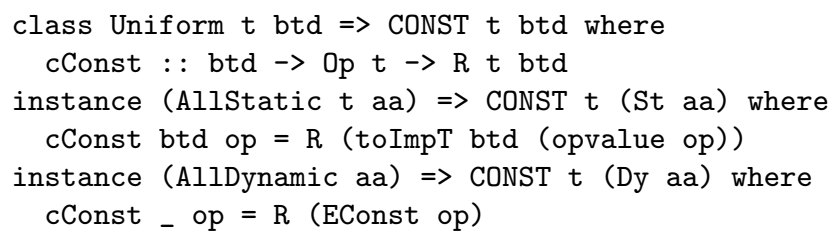

The dynamic case is straightforward, but the static case has a slight complication. Because of the recursive definition of ImpT for static bt descriptions, the type checker needs a proof that $t$ is equal to ImpT $t$ btd if btd is fully static. The class Uniform defines identities providing this proof in the usual way. ${ }^{5}$

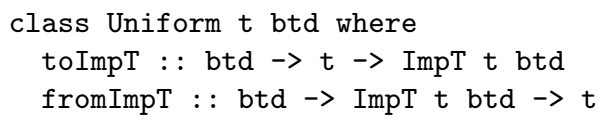

${ }^{5}$ See the appendix for the full definitions of Uniform, AllStatic, and AllDynamic. 


\section{$2.2 \quad$ Function Combinators}

The encoding of functions follows the ideas of higher-order abstract syntax as in previous work $[3,18,20]$. Thus, the generator represents bound variables by metavariables so that the cLam combinator for abstraction takes a function from representation type to representation type as an argument.

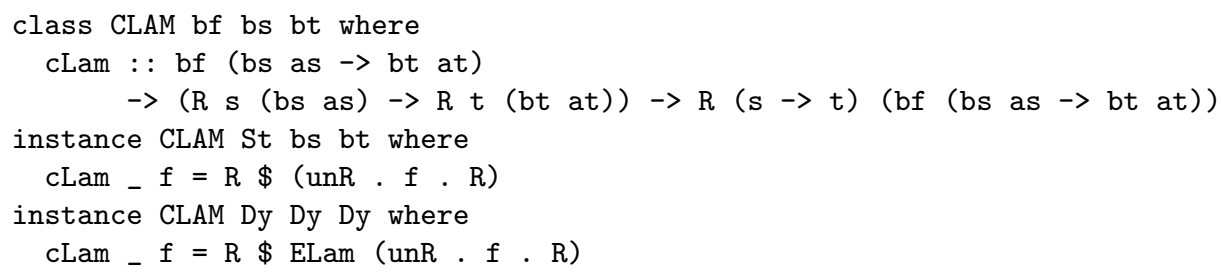

The two instances for the overloaded cLam combinator reflect exactly the bindingtime constraints: a static function does not restrict the binding time of its argument and result, whereas a dynamic function requires dynamic argument and result. The function ELam is the constructor for the typed (higher-order) abstract syntax (see Appendix A).

The definitions of the combinators cApp for function application and $\mathrm{CFix}$ for the fixpoint follow a similar scheme and are thus relegated to Appendix D.

\section{$2.3 \quad$ Subtyping}

Subtyping is the final important ingredient of binding-time analysis. This subtyping does not take part on the value level, but on the level of bt descriptions and expresses conversions between binding times. For example, a static integer can be converted into one of unknown binding time by the coercion (cSub (St int) ( $\mathrm{dz}$ int) ( $\mathrm{R}$ 1)) from Fig. 2.

In general, a coercion (cSub bfrom bto $v$ ) takes two bt descriptions and converts value $\mathrm{v}$ from binding time bfrom to binding time bto. The function cSub is defined in type class CSUB.

class CSUB b1 b2 $t$ where

cSub : : b1 $\rightarrow$ b2 $\rightarrow R$ t b1 $\rightarrow R$ t b2

The instances of this class follow the inductive definition of the subtyping relation in the binding-time analysis (see §3.4). For base types, it corresponds to the wellknown lifting operation.

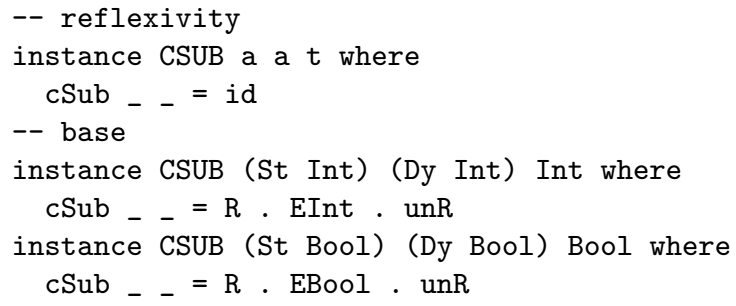

For function types, the code for the instances also follows the inductive definition, but it requires extra type annotations for technical reasons. Appendix E contains the full definitions. 


\subsection{List Operations}

Using the same principles as for functions, it is straightforward to develop combinators that support partially static operations on recursive data types. The signature for list-processing combinators serves as an example. Appendix F contains their implementations.

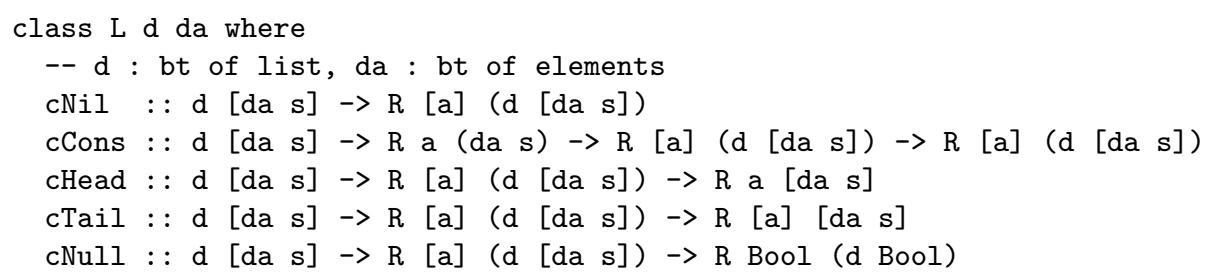

\section{From Binding-Time Analysis to Tagfree Program Generators}

This section defines a translation that maps a polymorphic binding-time type derivation generated by the polymorphic binding-time analysis of Glynn and coworkers [6] into a valid Haskell program that uses the combinators from $\S 2$. Precluding a formal correctness argument, we argue informally that the Haskell types express the binding-time constraints and, thus, that Haskell's type soundness guarantees specialization soundness. Furthermore, our automatic translation scheme relieves the programmer from the cumbersome task of writing tagfree program generators by hand.

Before we formalize the type-directed translation scheme, we recapitulate the essentials of Glynn's and coworkers polymorphic binding-time analysis and establish connections to our set of combinators.

\subsection{Underlying Type System}

We consider the translation of an ML-style let-polymorphic typed language with base types Int and Bool. For brevity, the formalization omits structured data types, but the implementation supports them $(\S 2.4)$.

$$
\begin{array}{ll}
\text { Types } & t::=\alpha \mid \text { Int } \mid \text { Bool } \mid t \rightarrow t \\
\text { Type Schemes } \sigma::=t \mid \forall \bar{\alpha} . t & \\
\text { Expressions } \quad e::=x|\lambda x . e| \text { ee } \mid \text { let } x=e \text { in } e
\end{array}
$$

The vector notation $\bar{\alpha}$ represents a sequence $\alpha_{1}, \ldots \alpha_{n}$ of type variables. Constructors for numerals and Boolean values are recorded in some initial type environment.

The treatment of Haskell's advanced language feature such as type classes is possible but postponed to future work. For instance, Glynn's and coworkers polymorphic binding-time analysis is performed on GHC's internal System F style type language CORE where type classes have already been 'removed' via the dictionary-passing translation. Hence, we would require combinators operating on GHC's CORE language directly to properly deal with type classes. 


$$
\Delta \vdash b: \text { Int } \quad \Delta \vdash b: \text { Bool } \quad \frac{(\beta: \alpha) \in \Delta}{\Delta \vdash \beta: \alpha} \quad \frac{\Delta_{1} \vdash \tau_{1}: t_{1} \quad \Delta_{2} \vdash \tau_{2}: t_{2}}{\Delta_{1} \cup \Delta_{2} \vdash \tau_{1} \stackrel{b}{\mapsto} \tau_{2}: t_{1} \rightarrow t_{2}}
$$

Fig. 4. Shape Rules

\subsection{Binding-Time Descriptions}

On top of the underlying type structure we impose a binding-time (type) description structure which reflects the structure of the underlying type system. For instance, $S \stackrel{S}{\mapsto} D$ describes a static function that takes a static value of base type as an argument and returns a dynamic value of base type.

$$
\begin{array}{ll}
\text { Annotations } & b::=\delta|S| D \\
\text { Binding-Time Descriptions } & \tau::=\beta|b| \tau \stackrel{b}{\mapsto} \tau \\
\text { Binding-Time Type Schemes } & \eta::=\tau \mid \forall \bar{\beta}, \bar{\delta} . C \Rightarrow \tau \\
\text { Constraints } & C::=(\tau \leq \tau)|w f t(\tau)| C \wedge C
\end{array}
$$

The grammar distinguishes annotation variables $\delta$, which may only be instantiated to $S$ or $D$, from binding-time type variables $\beta$, which may be instantiated to any $\tau$, including $\delta$. Constraints are described in $\S 3.4$.

\subsection{Shapes}

The binding-time description of an expression must generally have the same 'shape' as its underlying type, in particular in the presence of polymorphism. For this purpose, a shape environment $\Delta$ maps a polymorphic binding-time description variable to its corresponding underlying polymorphic type variable. The judgment $\Delta \vdash \tau: t$ states that under shape environment $\Delta$ the bindingtime description $\tau$ has shape $t$. A judgment $\Delta \vdash \eta: \sigma$ is valid if it can be derived by the shape rules in Figure 4. For brevity, we omit the straightforward rule for quantified types.

The combinator system in $\S 2$ detects ill-shaped types via unresolved instances. For example, the (ill-shaped) type $\mathrm{R}$ ( $\mathrm{a} \rightarrow \mathrm{b}$ ) (St Int) yields the unresolved type function application $\operatorname{ImpT}(\mathrm{a}->\mathrm{b})$ (St Int).

\subsection{Binding-Time Constraints}

A subtype constraint $(x \leq y)$ is read as " $y$ is at least as dynamic as $x$ ". It comes in various flavors: an ordering on annotations $\left(\cdot \leq_{a} \cdot\right)$, a structural ordering $\left(\cdot \leq_{s} \cdot\right)$ on bt descriptions, and an auxiliary ordering $\left(\cdot \leq_{f} \cdot\right)$, which is used in combination with the 'well-formed' constraint $w f t()$ to rule out 'ill-formed' constraints such as $S \stackrel{D}{\mapsto} S$. Figure 5 summarizes the constraint rules. 


$$
\begin{aligned}
& \text { (Sta) } C \vdash\left(S \leq_{a} b\right) \quad(\text { Dyn }) C \vdash\left(b \leq_{a} D\right) \\
& \text { (Нyp) } C_{1},\left(b_{1} \leq_{a} b_{2}\right), C_{2} \vdash\left(b_{1} \leq_{a} b_{2}\right) \\
& \text { (Refl) } C \vdash\left(b \leq_{a} b\right) \quad(\text { Trans }) \frac{C \vdash\left(b_{1} \leq_{a} b_{2}\right) C \vdash\left(b_{2} \leq_{a} b_{3}\right)}{C \vdash\left(b_{1} \leq_{a} b_{3}\right)} \\
& C \vdash\left(b_{3} \leq_{f} \tau_{1}\right) \quad C \vdash w f t\left(\tau_{1}\right) \\
& \left(\operatorname{Bas}_{w}\right) C \vdash w f t(b) \quad\left(\operatorname{Arrow}_{w}\right) \frac{C \vdash\left(b_{3} \leq_{f} \tau_{2}\right) \quad C \vdash w f t\left(\tau_{2}\right)}{C \vdash w f t\left(\tau_{1} \stackrel{b_{3}}{\longmapsto} \tau_{2}\right)} \\
& \left(\operatorname{Bas}_{f}\right) \frac{C \vdash\left(b_{1} \leq_{a} b_{2}\right)}{C \vdash\left(b_{1} \leq_{f} b_{2}\right)} \quad\left(\operatorname{Arrow}_{f}\right) \frac{C \vdash\left(b_{1} \leq_{a} b_{2}\right)}{C \vdash\left(b_{1} \leq_{f} \tau_{1} \stackrel{b_{2}}{\longmapsto} \tau_{3}\right)} \\
& \left(\operatorname{Bas}_{s}\right) \frac{C \vdash\left(b_{1} \leq_{a} b_{2}\right)}{C \vdash\left(b_{1} \leq_{s} b_{2}\right)} \quad\left(\text { Arrow }_{s}\right) \frac{C \vdash\left(\tau_{4} \leq_{s} \tau_{1}\right) C \vdash\left(\tau_{3} \leq_{s} \tau_{6}\right)}{C \vdash\left(\tau_{1} \stackrel{b_{2}}{\longmapsto} \tau_{3} \leq_{s} \tau_{4} \stackrel{b_{5}}{\longmapsto} \tau_{6}\right)}
\end{aligned}
$$

Fig. 5. Binding-Time Constraint Rules

Our combinator system detects ill-formed constraints via unresolved instances. For example, the irreducible constraint CLAM Dy St St corresponds to the illformed description $S \stackrel{D}{\mapsto} S$. A binding-time description is only well-formed if a dynamic annotation at the top of a binding-time description implies that all its components are dynamic, too, because nothing can be known about them. Hence, the above constraint is ill-formed.

The remaining binding-time subtype relations are expressed via the type class CSUB and its instances $(\S 2.3)$. The instance bodies construct the necessary coercions among binding-time values.

To be honest, the Haskell encoding leads to a slightly inferior system for the following reasons. First, the transitivity rule (Trans) cannot be easily expressed because the straightforward encoding in Haskell

instance (CSUB a $\mathrm{b} t, \operatorname{CSUB} \mathrm{b} c \mathrm{t}$ ) $\Rightarrow \operatorname{CSUB} a \mathrm{c}$

requires guessing the intermediate type $\mathrm{b}$ during type class instance resolution. A second short-coming of the Haskell encoding is that out of CSUB (St $(a->b)$ ) (St $(\mathrm{a} \rightarrow \mathrm{c})$ ) (Int $\rightarrow$ Int) we cannot extract the proof term (a.k.a. dictionary) connected to CSUB b c Int. The reverse direction is of course possible. Hence, if a program text requires CSUB (St $(\mathrm{a} \rightarrow \mathrm{b}))(\mathrm{St}(\mathrm{a}->\mathrm{c}))$ (Int $\rightarrow$ Int) but the surrounding context only provides CSUB b c Int, Haskell's type inference will fail. A simple workaround for both problems is to provide additional constraints which either mimic application of the transitivity rule or supply the necessary proof terms. 


\subsection{Type-Directed Translation from BTA to Program Generators}

Now everything is in place to describe the automatic construction of program generators based on our combinators out of Glynn and coworkers binding-time analysis. The construction is achieved via a type-directed translation scheme and relies on judgments of the form $C, \Gamma \vdash(e:: t): \tau \rightsquigarrow\left(e_{H} \mathbf{I} C_{H}\right)$ where $C$ is a binding-time constraint, $\Gamma$ a binding-time environment, $e$ an expression welltyped in the underlying system with type $t, \tau$ is a binding-time description, $e_{H}$ is the Haskell expression derived from $e$ instrumented with program generator combinators and $C_{H}$ is a Haskell constraint which contains all the requested combinator instances including subtype (coercion) constraints.

Figure 6 contains the (non-syntax directed) translation rules. It is an easy exercise to make them syntax directed, following Glynn and coworkers [6].

In rule (Sub), $C \rightsquigarrow C_{H}$ denotes the translation of binding-time subtype constraints $\left(\tau_{1} \leq \tau_{2}\right)$ to Haskell type class constraints CSUB $\tau_{1} \tau_{2} t$ for some appropriate $t .{ }^{6}$ Ill-formed binding-time descriptions are caught via unresolved instances. Hence, the translation simply drops the well-formed constraint $w f t(\tau)$. The translation of the judgment $C \vdash\left(\tau_{2} \leq_{s} \tau_{1}\right)$ to the Haskell setting may not hold any more, unless $C$ contains redundant constraints as discussed in $\S 3.4$. Hence, we assume from now on that such redundant constraints are present in $C$. In the resulting (Haskell) program text, the expression $e_{H}$ is coerced to the expected bt description $\tau_{1}$ by inserting the combinator call cSub $\tau_{2} \tau_{1}$. Descriptions such as $\tau_{1}$ occurring in expressions are short-hands for undefined $:: \tau_{1}$ where variables appearing in $\tau_{1}$ are bound by lexically scoped type annotations. ${ }^{7}$ Recall that binding-time descriptions passed at run-time are never inspected. Thanks to laziness they have virtually no cost.

Rule (Abs) and (App) are straightforward and do not contain any surprises. The rule (Let) additionally abstract over the binding-time descriptions $\bar{\beta}$ and $\bar{\delta}$ which then will be supplied with arguments at the instantiation site (see rule $(\forall \mathrm{E})$ ). The function inst computes the corresponding binding-time description instances for each underlying type instance. Let $\Delta$ be a shape environment, $\bar{t}$ a sequence of underlying types, and $\bar{\alpha}$ a sequence of underlying type variables. Let inst $(\Delta, \bar{t}, \bar{\alpha})=\bar{\tau}$ where $\bar{\tau}$ are fresh binding-time types of appropriate shape: Each element of $\bar{\tau}$ is related to the corresponding element of $\bar{\beta}$ by the shape environment $\Delta$. That is, $\Delta, t_{i} \vdash \tau_{i j}$ where $\Delta \vdash \beta_{i j}: \alpha_{i}$.

The last rule (Fix) deals with polymorphic recursion (in the binding-time descriptions). A fixpoint iteration is required to compute the set of combinator instances CIF etc. The constraints resulting from $(e:: t)$ are split into those, which are not connected to $\bar{\delta}\left(C_{1 H}\right)$, and those which constrain $\bar{\delta}\left(C_{2 H}\right)$. The fixpoint operator $\mathcal{F}$ starts with $C_{2 H}$ plus the Haskell equivalent $C_{2}^{\prime}{ }_{H}$ of the subtype constraints in $C_{2}$ and iterates until a fixpoint $C_{3 H}$ is found. The exact

\footnotetext{
${ }^{6}$ In a syntax-directed inference system the program text determines the type $t$.

7 The alternative is to build an explicit term of type $\tau_{1}$ as in Fig. 2.
} 
definition of $\mathcal{F}$ is as follows:

$$
\begin{aligned}
& \mathcal{F}\left(\Gamma \cup\left\{x: \forall \bar{\delta} . C_{2 H} \Rightarrow \tau\right\}, e:: t\right) \\
& =\mathcal{F}\left(\Gamma \cup\left\{x: \forall \bar{\delta} . C_{2 H} \wedge C_{3 H} \Rightarrow \tau\right\}, e:: t\right) \quad \text { if } C_{2 H} \neq_{s e t} C_{3 H} \\
& =C_{2 H} \quad \text { otherwise }
\end{aligned}
$$

where $\Gamma \cup\left\{x: \forall \bar{\delta} . C_{2 H} \Rightarrow \tau\right\} \vdash(e:: t): \tau \rightsquigarrow\left(e_{H} \mathbf{I} C_{3 H}\right)$.

The following example serves to illustrate the fixpoint iteration.

$f x y=$ if $x=0$ then 1 else $f y(x-1)$

Function $f$ 's most general binding time description is

$$
\forall b_{x}, b_{y}, b .\left(b_{x} \leq b\right) \wedge\left(b_{y} \leq b\right) \Rightarrow b_{x} \stackrel{S}{\mapsto} b_{y} \stackrel{S}{\mapsto} b
$$

Binding-time polymorphism is required in the subexpression $f y(x-1)$ for building the instance $b_{y} \stackrel{S}{\mapsto} b_{x} \stackrel{S}{\mapsto} b$.

The first step of the translation yields the following (Haskell) constraints from the program text:

SUB bx b Int, SUB by $b$ Int,

CIF bx b,

CLAM St by $b$, CLAM St bx (by->b),

CAPP St $b x b$, CAPP St by $(b x->b)$

These constraints are not sufficient for the resulting program to type check. For example, at the instantiation site $f$ y $(x-1)$ the constraint CIF by $b$ is needed but there is only CIF bx b. Another iteration starting with the above constraints leads to the fixpoint: ${ }^{8}$

SUB bx b Int, SUB by $b$ Int,

CIF bx b, CIF by b,

CLAM St by $b$, CLAM St $b x(b y->b)$, CLAM St $b x b$, CLAM St by $(b x->b)$,

CAPP St bx b, CAPP St by $(b x->b)$, CAPP St by b, CAPP St bx (by->b)

The fixpoint iteration must terminate because it only iterates over annotations whose shape is fixed/bound by the underlying type. Hence, the number of instances arising is finite.

An alternative translation scheme could employ the cFix combinator also provided by the library. It corresponds to a monomorphic (Fix) rule, which requires no fixpoint iteration.

In summary, the type-directed translation scheme builds a tight correspondence between the typing of the combinators and the typing rules of the bindingtime analysis. It might be stated as a slogan in the following way.

Proposition 1. Let True, $\emptyset \vdash(e:: t): \tau \rightsquigarrow\left(e_{H} \mathbf{I} C_{H}\right)$. Then, the resulting expression $e_{H}$ is well-typed in Haskell with type $R t \tau$ under constraints $C_{H}$.

\footnotetext{
$\overline{8}$ The fixpoint iteration requires a variant of the $(\forall \mathrm{E})$ rule which also infers the required instantiation constraints, rather than simply checking if the provided constraints imply the instantiation constraints. For reasons of space, we omit the straightforward details.
} 
We have no proof for this proposition, although it is easy in many cases to match the typing of a single combinator with its corresponding typing rule. An attempt to prove it would have to overcome the shortcomings discussed in the preceding text and it would have to draw on a formalization of a large subset of Haskell's type system. Both tasks are out of scope of the present work.

\section{Related Work and Conclusion}

Among the large body of related work on partial evaluation (see the respective overviews $[7,11]$ ), there are only few works which consider offline partial evaluation based on a polymorphic binding-time analysis for polymorphic languages $[6,8,9]$. None of them consider the direct construction of program generators. Only Heldal and Hughes [8] deal with the pragmatics of constructing the specializer. Other works that consider polymorphism concentrate either on polymorphic binding-time analysis for monomorphic languages [5, 10] or monomorphic analysis for polymorphic languages $[4,12,14]$.

Closely related are previous constructions of combinators that perform specialization by the first author $[18,20]$ as well as combinators by Carette and coworkers [3] that can be statically configured (either via overloading or via the ML module language) to perform evaluation, compilation, or (online) partial evaluation. Two main differences to the latter work are (1) that our combinators are geared towards offline partial evaluation and require a preceding bindingtime analysis and (2) that our combinators are dynamically configured by type passing.

The present work complements the earlier work of Glynn and coworkers [6] and puts it into practice. Our combinators solve the open question of obtaining safe and efficient (tag-free) program generators for ML-style languages based on a polymorphic binding-time analysis. Our proof-of-concept implementation relies on GHC's advanced (source) typing features and allows us to experiment with smaller examples.

There are many opportunities for future work. We doubt that there an analogous set of combinators that can be implemented in ML, but it is an interesting question to consider. We believe that the approach is extensible to typing features of Haskell beyond ML. We further believe that the approach can be extended to cater for typical partial evaluation features like program point specialization, multi-level specialization, or continuation-based specialization.

\section{References}

1. L. Birkedal and M. Welinder. Hand-writing program generator generators. In M. V. Hermenegildo and J. Penjam, editors, Intl. Symp. Programming Languages, Implementations, Logics and Programs (PLILP '94), volume 844 of LNCS, pages 198-214, Madrid, Spain, Sept. 1994. Springer.

2. A. Bondorf and D. Dussart. Improving CPS-based partial evaluation: Writing cogen by hand. In P. Sestoft and H. Søndergaard, editors, Proc. 1994 ACM Workshop Partial Evaluation and Semantics-Based Program Manipulation, pages 1-10, 
Orlando, Fla., June 1994. University of Melbourne, Australia. Technical Report 94/9, Department of Computer Science.

3. J. Carette, O. Kiselyov, and C. chieh Shan. Finally tagless, partially evaluated: Tagless staged interpreters for simpler typed languages. J. Funct. Program., 19(5):509$543,2009$.

4. A. De Niel, E. Bevers, and K. De Vlaminck. Partial evaluation of polymorphically typed functional languages: the representation problem. In JTASPEFT/WSA'91, pages 90-97, 1991.

5. D. Dussart, F. Henglein, and C. Mossin. Polymorphic recursion and subtype qualifications: Polymorphic binding-time analysis in polynomial time. In A. Mycroft, editor, Proc. 1995 International Static Analysis Symposium, volume 983 of LNCS, pages 118-136, Glasgow, Scotland, Sept. 1995. Springer.

6. K. Glynn, P. Stuckey, M. Sulzmann, and H. Søndergaard. Boolean constraints for binding-time analysis. In Programs as Data Objects II, volume 2053 of LNCS, pages 39-62, Aarhus, Denmark, May 2001. Springer.

7. J. Hatcliff, T. Æ. Mogensen, and P. Thiemann, editors. Partial EvaluationPractice and Theory. Proceedings of the 1998 DIKU International Summerschool, volume 1706 of LNCS, Copenhagen, Denmark, 1999. Springer.

8. R. Heldal and J. Hughes. Binding-time analysis for polymorphic types. In PSI-01: Andrei Ershov Fourth International Conference, Perspectives of System Informatics, volume 2244 of $L N C S$, pages 191-204, Novosibirsk, Russia, July 2001. Springer.

9. S. Helsen and P. Thiemann. Polymorphic specialization for ML. ACM TOPLAS, 26(4):1-50, July 2004.

10. F. Henglein and C. Mossin. Polymorphic binding-time analysis. In D. Sannella, editor, Proc. 5th ESOP, volume 788 of LNCS, pages 287-301, Edinburgh, UK, Apr. 1994. Springer.

11. N. Jones, C. Gomard, and P. Sestoft. Partial Evaluation and Automatic Program Generation. Prentice-Hall, 1993.

12. J. Launchbury. A strongly-typed self-applicable partial evaluator. In J. Hughes, editor, Proc. FPCA 1991, volume 523 of LNCS, pages 145-164, Cambridge, MA, USA, 1991. Springer.

13. J. Launchbury and C. K. Holst. Handwriting cogen to avoid problems with static typing. In Draft Proceedings, Fourth Annual Glasgow Workshop on Functional Programming, pages 210-218, Skye, Scotland, 1991. Glasgow University.

14. T. Æ. Mogensen. Binding time analysis for polymorphically typed higher order languages. In J. Díaz and F. Orejas, editors, TAPSOFT '89, volume 351,352 of LNCS, pages II, 298-312, Barcelona, Spain, Mar. 1989. Springer.

15. S. Peyton Jones, editor. Haskell 98 Language and Libraries, The Revised Report. Cambridge University Press, 2003.

16. S. Peyton Jones, D. Vytiniotis, S. Weirich, and M. Shields. Practical type inference for arbitrary-rank types. J. Funct. Program., 17(1):1-82, 2007.

17. T. Schrijvers, S. L. Peyton Jones, M. M. T. Chakravarty, and M. Sulzmann. Type checking with open type functions. In P. Thiemann, editor, Proc. ICFP 2008, pages 51-62, Victoria, BC, Canada, Oct. 2008. ACM Press, New York.

18. P. Thiemann. Cogen in six lines. In K. Dybvig, editor, Proc. 1996 ICFP, pages 180-189, Philadelphia, PA, May 1996. ACM Press, New York.

19. P. Thiemann. Aspects of the PGG system: Specialization for standard Scheme. In Hatcliff et al. [7], pages 412-432.

20. P. Thiemann. Combinators for program generation. J. Funct. Program., 9(5):483525, Sept. 1999. 
(Var)

$$
\frac{(x: \eta) \in \Gamma \quad \Delta \vdash \eta: \sigma \quad C \rightsquigarrow C_{H}}{C, \Gamma \vdash(x:: \sigma): \eta \rightsquigarrow\left(x \mathbf{I} C_{H}\right)}
$$

(Sub) $\frac{C, \Gamma \vdash(e:: t): \tau_{2} \rightsquigarrow\left(e_{H} \mathbf{I} C_{H}\right) \quad C \vdash\left(\tau_{2} \leq_{s} \tau_{1}\right) \quad C \vdash w f t\left(\tau_{1}\right)}{C, \Gamma \vdash(e:: t): \tau_{1} \rightsquigarrow\left(\operatorname{cSub} \tau_{2} \tau_{1} e_{H} \mathbf{I} C_{H} \wedge \operatorname{CSUB} \tau_{2} \tau_{1} t\right)}$

$$
\begin{aligned}
& C, \Gamma \cup\left\{x: \tau_{1}\right\} \vdash\left(e:: t_{2}\right): \tau_{2} \rightsquigarrow\left(e_{H} \mathbf{I} C_{H}\right) \\
& \frac{C \vdash w f t\left(\tau_{1}\right) \Delta \vdash \tau_{1}: t_{1}}{C, \Gamma \vdash\left(\lambda x . e:: t_{1} \rightarrow t_{2}\right): \tau_{1} \stackrel{S}{\mapsto} \tau_{2}} \\
& \left.\left(\operatorname{cLam} \operatorname{ST}\left(\tau_{1} \mapsto \tau_{2}\right)\left(\lambda x . e_{H}\right) \mathbf{I} C_{H} \wedge \operatorname{CLAM~ST} \tau_{1} \tau_{2}\right\}\right) \\
& C_{1}, \Gamma \vdash\left(e_{1}:: t_{1} \rightarrow t_{2}\right):\left(\tau_{1} \stackrel{b}{\mapsto} \tau_{2}\right) \rightsquigarrow\left(e_{1 H} \mathbf{I} C_{1 H}\right) \\
& \begin{array}{c}
C_{2}, \Gamma \vdash\left(e_{2}:: t_{1}\right): \tau_{1} \rightsquigarrow\left(e_{2 H} \mid C_{2 H}\right) \\
C_{1} \wedge C_{2}, \Gamma \vdash\left(e_{1} e_{2}:: t_{2}\right): \tau_{2}
\end{array}
\end{aligned}
$$

$\left(\operatorname{cApp}\left(b\left(\tau_{1} \mapsto \tau_{2}\right)\right) e_{H_{H}} e_{2 H} \mathbf{I} C_{1 H} \wedge C_{2 H} \wedge \operatorname{CAPP} b \tau_{1} \tau_{2}\right)$

$$
\begin{gathered}
C_{1}, \Gamma \vdash\left(e_{1}:: t_{1}\right): \tau_{1} \rightsquigarrow\left(e_{1 H} \mathbf{I} C_{1 H}\right) \\
\Delta \vdash \tau_{1}: t_{1} \quad \bar{\beta}, \bar{\delta} \subseteq f v\left(C_{1}, \tau_{1}\right) \backslash f v(\Gamma) \\
\text { where } \Delta \vdash \beta_{i j}: \alpha_{i}
\end{gathered}
$$

(Let) $\frac{C_{2}, \Gamma \cup\left\{x: \forall \bar{\beta} \bar{\delta} . C_{1} \Rightarrow \tau_{1}\right\} \vdash\left(e_{2}:: t_{2}\right): \tau_{2} \rightsquigarrow\left(e_{2 H} \mathbf{I} C_{2 H}\right)}{C_{2}, \Gamma \vdash\left(\text { let } x=\left(e_{1}:: \forall \bar{\alpha} . t_{1}\right) \text { in } e_{2}:: t_{2}\right): \tau_{2}}$

$$
\rightsquigarrow
$$

(let $x=\left(\lambda \bar{\beta} \cdot \lambda \bar{\delta} \cdot e_{1_{H}}:: \forall \bar{\alpha} \bar{\beta} \bar{\delta} \cdot C_{1_{H}} \Rightarrow R t_{1} \tau_{1}\right)$ in $\left.e_{2 H} \mathbf{I} C_{2_{H}}\right)$

$$
C, \Gamma \vdash(e:: \forall \bar{\alpha} . t): \forall \bar{\beta}, \bar{\delta} . D \Rightarrow \tau \rightsquigarrow\left(e_{H} \mathbf{I} C_{H}\right)
$$

$(\forall \mathrm{E})$

$$
\Delta \vdash \tau: t \quad \operatorname{inst}(\Delta, \bar{t}, \bar{\alpha})=\bar{\tau}
$$

$$
\frac{C \vdash[\bar{\tau} / \bar{\beta}, \bar{b} / \bar{\delta}] D}{C, \Gamma \vdash(e::[\bar{t} / \bar{\alpha}] t):[\bar{\tau} / \bar{\beta}, \bar{b} / \bar{\delta}] \tau \rightsquigarrow\left(e_{H} \bar{\tau} \bar{b} \mathbf{I} C_{H}\right)}
$$

$$
\eta=\forall \bar{\delta} \cdot C_{2} \Rightarrow \tau \quad C_{2} \rightsquigarrow C_{2}^{\prime}
$$

$C_{1} \wedge C_{2}, \Gamma \cup\{x: \eta\} \vdash(e:: t): \tau \rightsquigarrow\left(e_{H} \mathbf{I} C_{1 H} \wedge C_{2 H}\right)$

$C_{1} \wedge C_{2} \vdash w f t(\tau) \quad \Delta \vdash \eta: t$

(Fix)

$$
f v\left(C_{1_{H}}\right) \cap \bar{\delta}=\emptyset \quad f v\left(C_{2 H}\right) \subseteq \bar{\delta}
$$

$\frac{\mathcal{F}\left(\Gamma \cup\left\{x: \forall \bar{\delta} . C_{2}{ }_{H}^{\prime} \wedge C_{2 H} \Rightarrow \tau\right\}, e:: t\right)=C_{3 H}}{C_{1}, \Gamma \vdash((\text { fix } x:: t \text { in } e):: t): \eta}$

(let $x=\left(\lambda \bar{\delta} . e_{H}:: \forall \bar{\delta} \cdot C_{3 H} \Rightarrow R t \tau\right)$ in $\left.x \mathbf{I} C_{1_{H}}\right)$

Fig. 6. Type-direction translation rules 
This appendix contains additional material for scrutiny by the interested reviewer. The source code of the combinators is available online at http://proglang.informatik.uni-freiburg.de/projects/polyspec/

\section{A Expression Datatype}

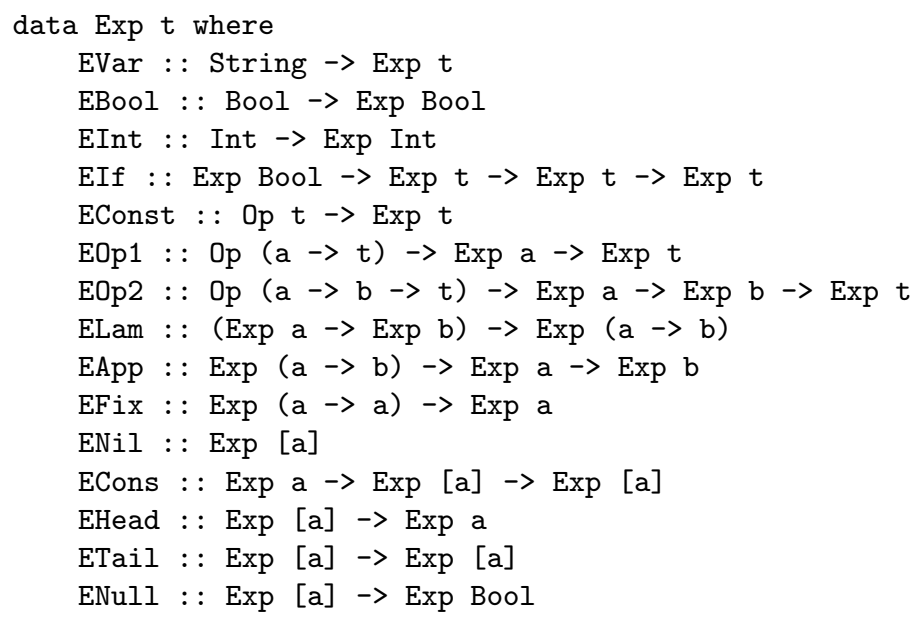




\section{B Uniform Binding-Time Descriptions}

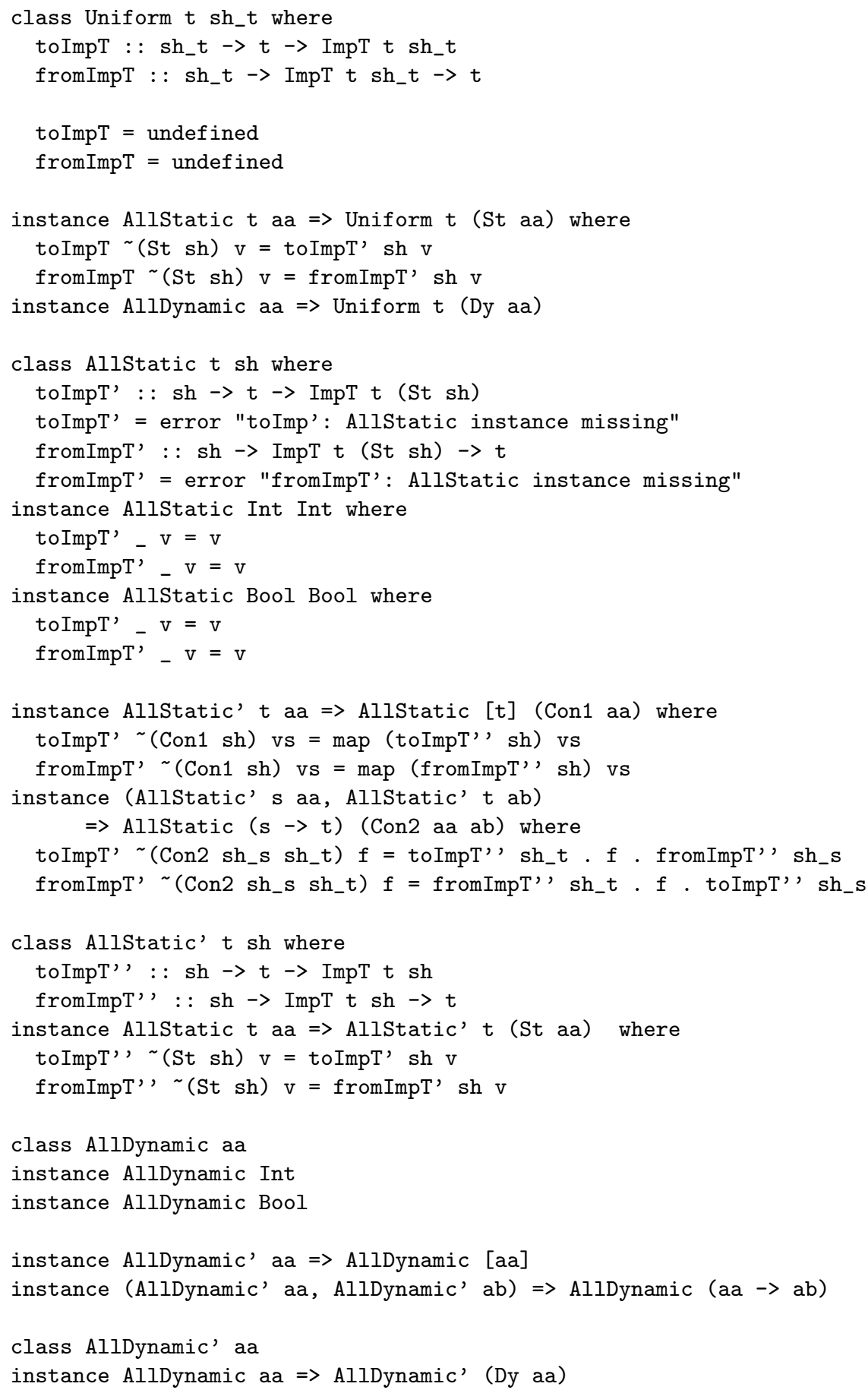




\section{Primitive Operators}

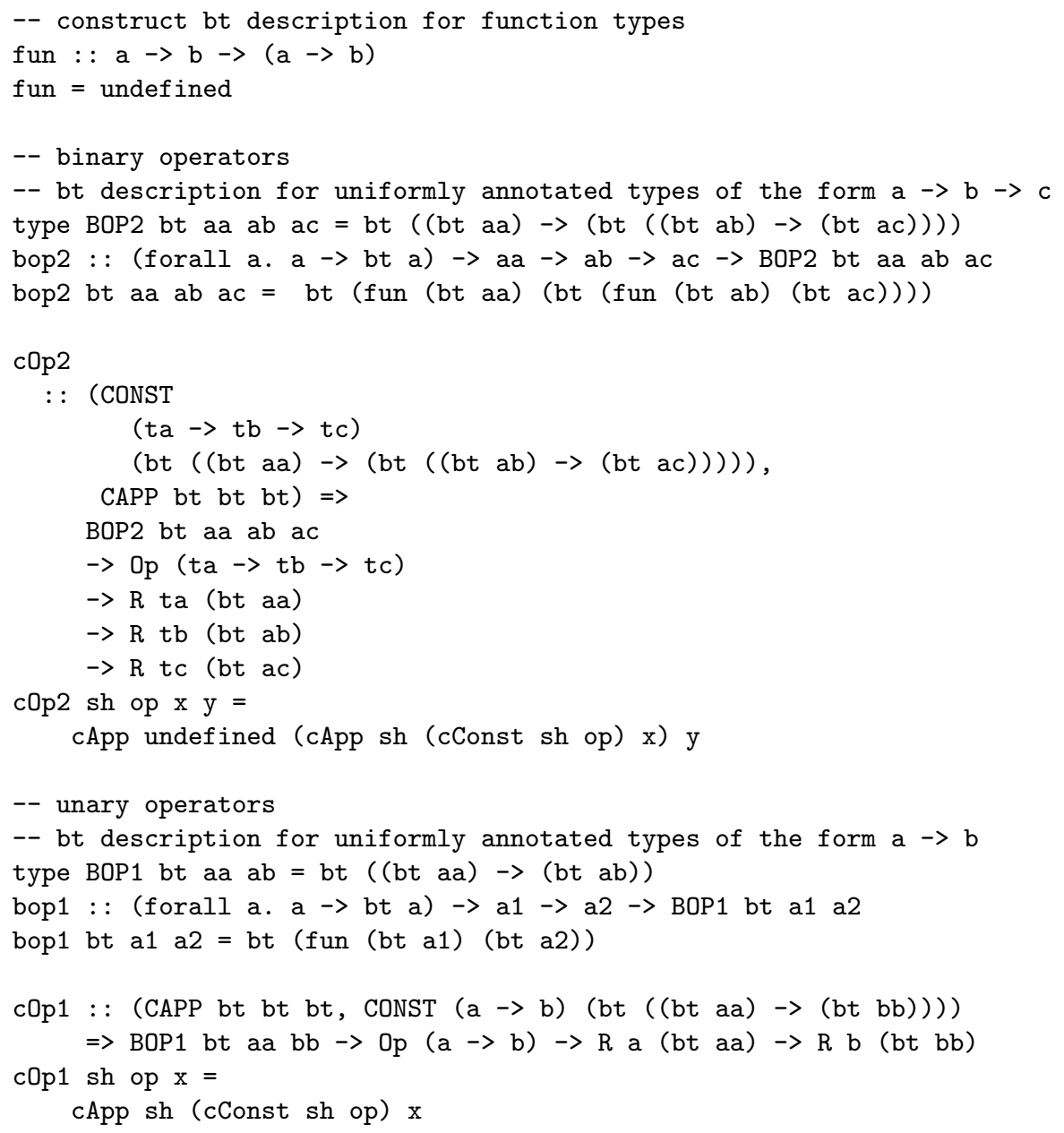




\section{Function Application and Fixpoint}

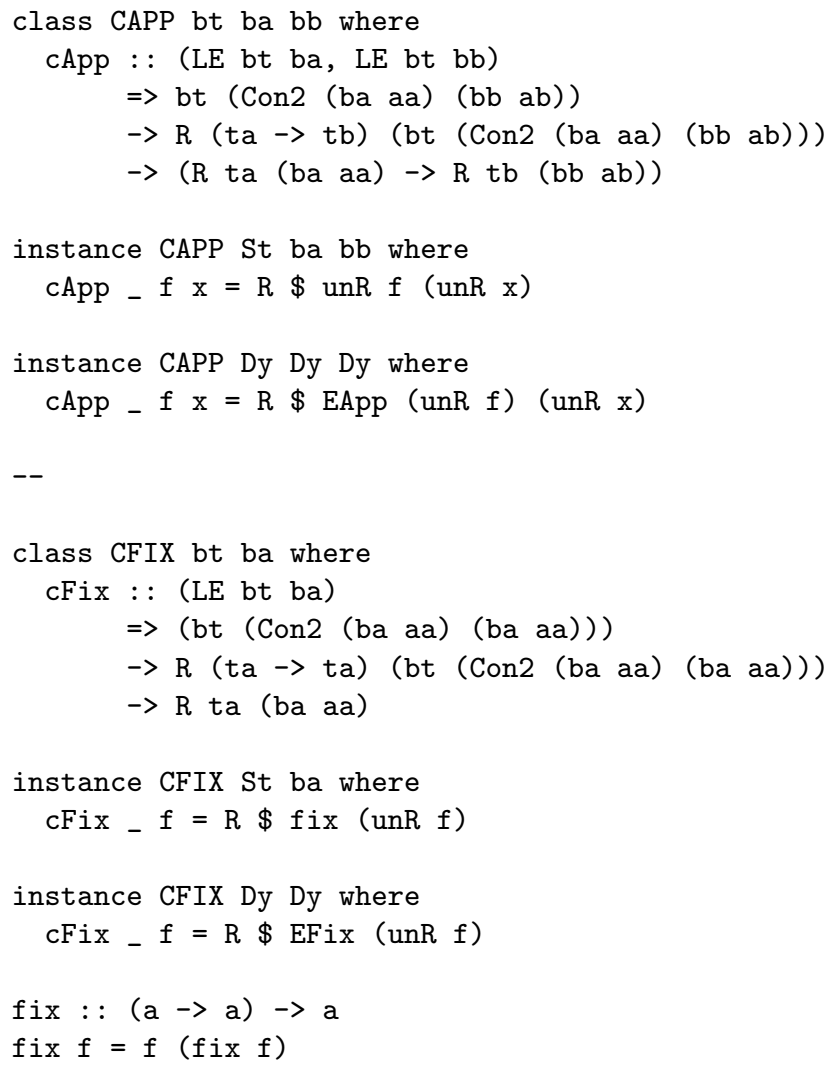




\section{E Subtyping for Functions}

\section{E.1 Static Functions}

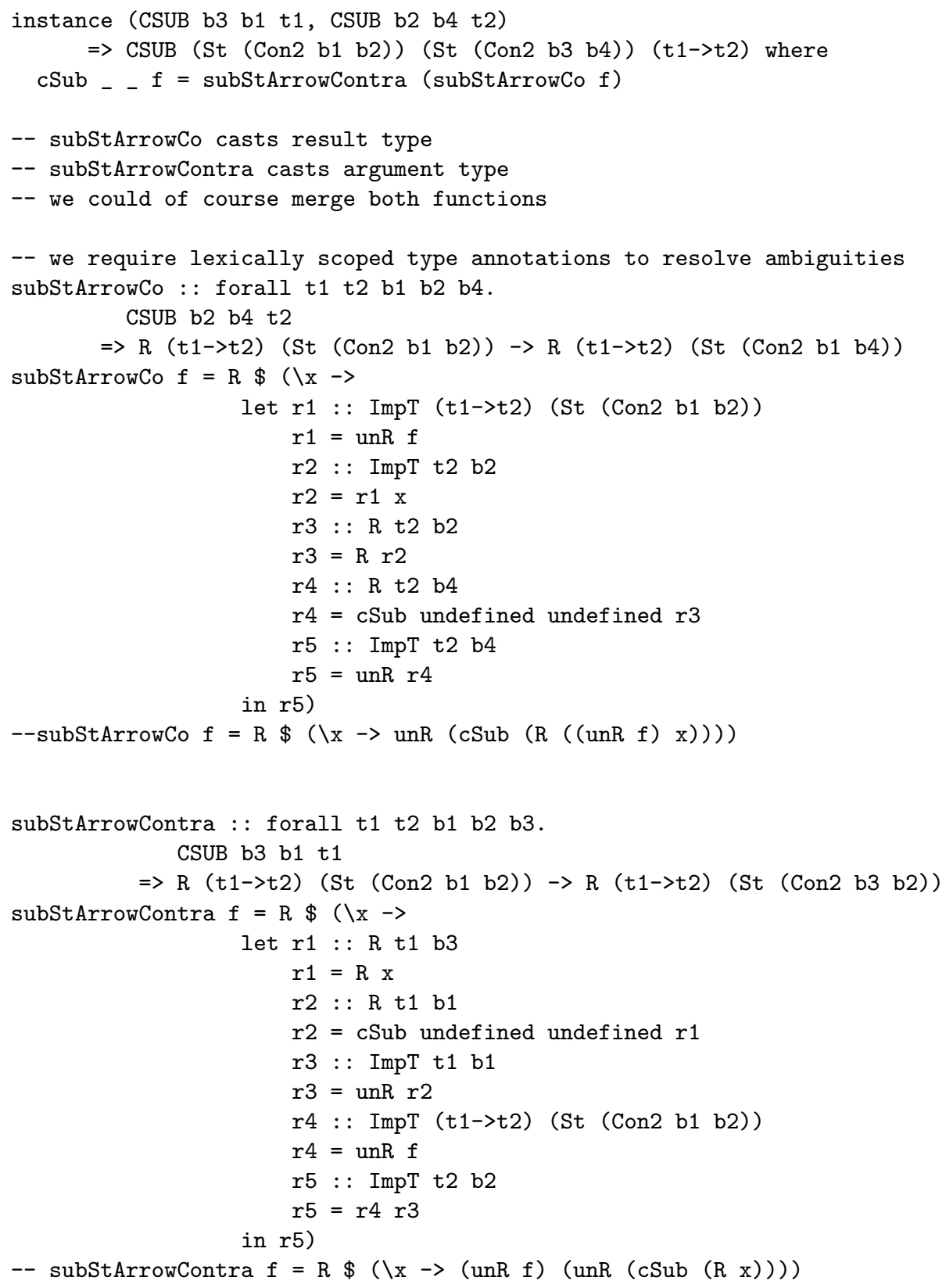




\section{E.2 Dynamic Functions}

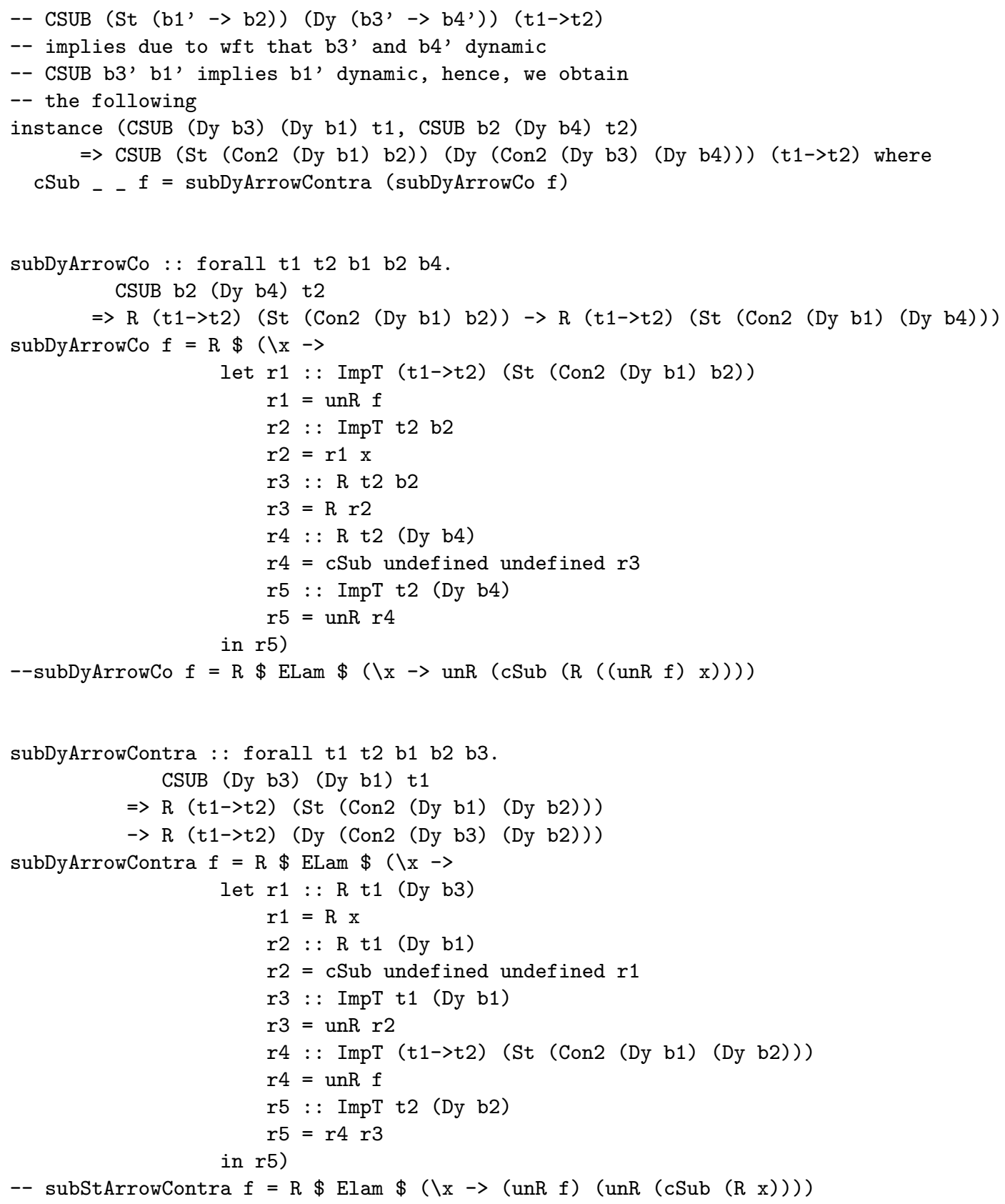




\section{F List Processing}

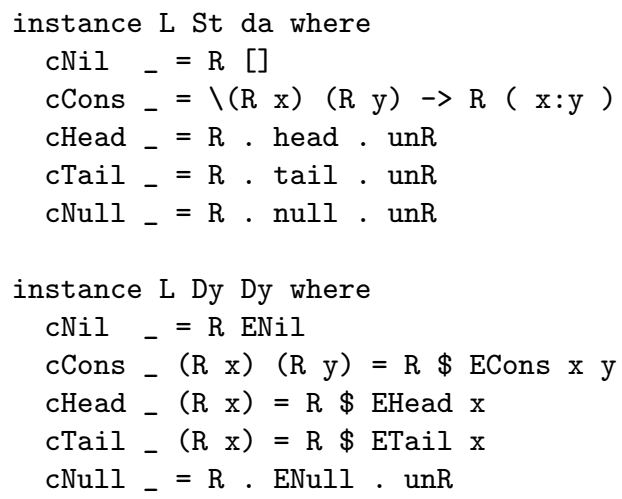

